five distinct sources and on material specially pre pared in the Forest Products Research Laboratory.

Starch.-The corresponding results which we have obtained with starch vary according to several factors : for example, the nature of the amylose used and whether the material is methylated directly or is subjected to a preliminary acetylation. When the hydrolysis of methylated amylose is carried out in stages, tetramethyl glucose can be detected in amounts varying from 3 to nearly 5 per cent; but of much greater importance is the observation that one fraction of the methylated amylose is convertible into a mixture of sugars, the composition of which varied within the following limits :

$2: 3: 4: 6$-Tetramethyl glucose

$2: 3: 6$-Trimethyl glucose

2 : 3- and $2: 6$-Dimethyl glucose.

23-26 per cent

55-52 per cent

21 per cent

This indicates that the group responsible for the formation of tetramethyl glucose forms part of a complicated fragment of the amylose complex, as otherwise no dimethyl glucose would be formed from a fully methylated derivative. We have shown that this dimethyl glucose is a mixture of $2: 3$ - and $2: 6$-isomerides in approximately equal proportions when the amylose has been methylated directly; but this ratio is disturbed to 24 and 76 per cent respectively as the result of preliminary acetylation. This, in itself, indicates the extreme caution which must be exercised in applying the methylation method in conjunction with acetylation to polysaccharides.

Inulin.-Trimethyl inulin when hydrolysed with oxalic acid yields, in addition to trimethyl $\gamma$-fructoses, a mixture of (a) w-methoxy-5-methylfurfural, (b) tetramethyl $\gamma$-fructose and $(c)$ a trimethyl anhydrofructose. We find that the yields of tetramethyl $\gamma$-fructose have varied from $2 \cdot 7$ as a maximum to 1.7 per cent as a minimum, owing to partial conversion of the sugar into the furfural derivative. If all of the above products be calculated as tetramethyl fructose, inulin would consist of a chain of 25 fructose units; whilst taking the above yield of 2.7 per cent as a basis, the inulin molecule would contain 43 fructose units in the chain.

We have encountered so many complications in our studies of methylated polysaccharides (including the unexpected result that certain $\gamma$-fructose derivatives are readily transformed into derivatives of glucose) that we are reluctant to make far-reaching claims on the basis of having isolated such compounds as tetramethyl glucose and tetramethyl fructose under the conditions described; but this communication will serve to show the progress we have made recently in researches which were initiated in this laboratory and have not been discontinued.

J. C. Irvine.

Chemical Research Laboratory,

The University, St. Andrews, March 12.

\section{Vertical Tube Counter- and the Barometric-Effect of Cosmic Radiation at Sea-level}

TURNING a long tube counter in a perpendicular plane, the number of recorded cosmic ray particles depends on the angle $a$ between the vertical direction and the axis of the tube. This vertical tube counter effect, first observed by Tuwim ${ }^{x}$ at Potsdam, has now been measured more precisely and found to be in accordance with his theory of this phenomenon.

The number $N_{\mu H}\left(a^{\circ}\right)$ is proportional to $\sin ^{2} a$, as shown in Fig. 1. This straight line enables the elimination of experimental errors of the single measurements, and therefore the fraction $N_{\mu H}\left(0^{\circ}\right): N_{\mu_{H}}\left(90^{\circ}\right)$ and the mean mass absorption coefficient of the cosmic radiation can be found more exactly than before. In a lead screen, $10 \mathrm{~cm}$. thick, the coefficient was found to be $(\mu / \rho)_{\mathrm{H}_{2} \mathrm{O}}=8.01$ and $8.12 \times 10^{-4} \mathrm{~cm} .^{2} \mathrm{gm}^{-1}$ with the two tube counters No. 21 and No. $22(r=2 \cdot 4$, $l=45.0 \mathrm{~cm}$. and $46.0 \mathrm{~cm}$.) respectively, in accordance with Tuwim's value $(9 \pm 1) \times 10^{-4} \mathrm{~cm} .{ }^{2} \mathrm{gm} \cdot .^{-1}$ obtained with tube counter No. $15(r=2 \cdot 4, l=15 \cdot 3 \mathrm{~cm}$.).

Repeating the experiments in the open air at different barometric pressures, the straight line of the vertical tube counter efiect is shifted parallel, showing thus the barometric effect ${ }^{2}$ very clearly. Assuming a pure absorption phenomenon for this behaviour, ${ }^{3}$ the mean absorption coefficient is $(\mu / \rho)_{\mathrm{H}_{2} \mathrm{O}}=1.67 \times 10^{-3}$ $\mathrm{cm} .^{2} \mathrm{gm} .^{-1}$. From the vertical tube counter effect it was found to be $(\mu / \rho)_{\mathrm{H}_{2} \mathrm{O}}=1.69 \times 10^{-3} \mathrm{~cm} .^{2} \mathrm{gm} .^{-1}$ in the open air.

By two different methods, therefore, it is possible to measure accurately the absorption coefficient of cosmic

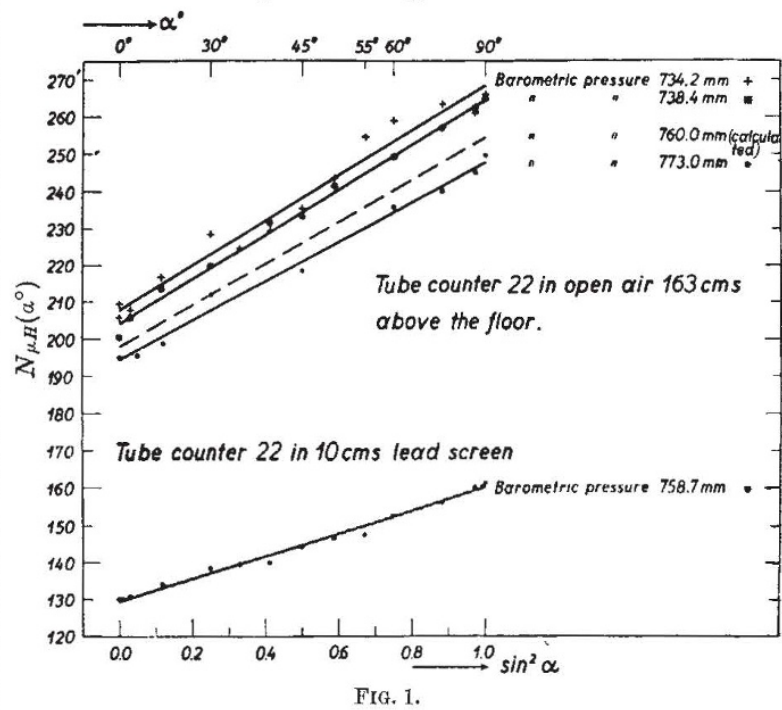

radiation with the same apparatus and at the same time, but without a special screen indispensable for the usual method of determining absorption coefficients.

The accordance of the two values for the open air confirms the theory of the vertical tube counter effect, and should be interpreted by this theory that the direction of the cosmic rays, acting in the counter, is not greatly different from the initial direction of the rays entering the atmosphere. The conflicting results of Skobeltzyn ${ }^{4}$ with the Wilson chamber are therefore probably due to the greater statistical uncertainty of his measurements ; for, according to him, no vertical tube counter effect of the cosmic radiation can exist.

W. KoLHÖRSTER.

Höhenstrahlungslaboratorium des

Meteorologisch-Magnetischen Observatoriums, Potsdam, Jan. 31.

1 L. Tuwim, Berlin, Ber. 91, 360 ; 1931

2 L. Myssowsky and L. Tuwim, Z. Physik, 39, 146; 1926.

s W. Kolhörster and L. Tuwim, Erg. kosm. Physik, 1, 127; 1931.

4 D. Skobeltzyn, C.R., 194, 118; 1932

\section{Molecular Refraction of Nitrobenzene}

The discontinuities observed by Mazur in certain physical properties of nitrobenzene in the neighbourhood of $9.5^{\circ} \mathrm{C}$., and described in a series of communications to NATURE, call for further investigation. The sharp decrease in the dielectric constant ${ }^{1}$ at $9 \cdot 6^{\circ} \mathrm{C}$., the distinct retardation in the heating curve ${ }^{2}$ at $9.5^{\circ} \mathrm{C}$., and the change in slope of the density- 